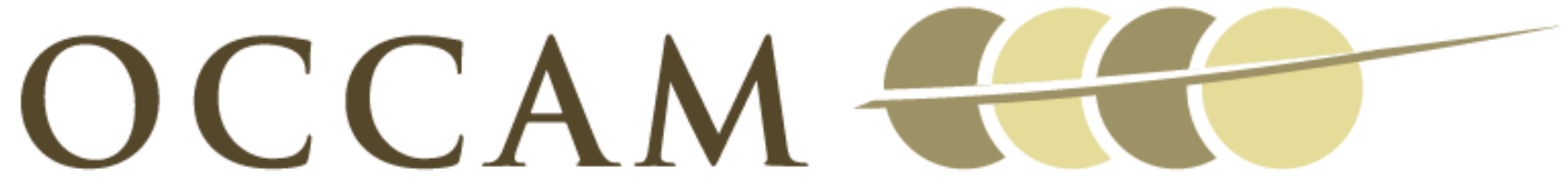

OXFORD CENTRE FOR COLLABORATIVE APPLIED MATHEMATICS

Report Number 10/35

Regularized Particle Filter with Langevin Resampling Step

by

Lian Duan, Chris L. Farmer, and Irene M. Moroz

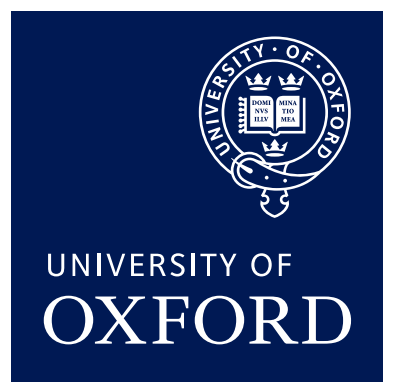

Oxford Centre for Collaborative Applied Mathematics Mathematical Institute

24 - 29 St Giles'

Oxford

OX1 3LB

England 



\title{
Regularized Particle Filter with Langevin Resampling Step
}

\author{
Lian Duan*, Chris L. Farmer* and Irene M. Moroz ${ }^{\dagger}$ \\ * Oxford Centre for Collaborative Applied Mathematics, Mathematical Institute, University of Oxford, OX1 3LB \\ $\dagger$ Oxford Centre for Industrial and Applied Mathematics, Mathematical Institute, University of Oxford, OX1 3LB
}

\begin{abstract}
The solution of an inverse problem involves the estimation of variables and parameters values given by the statespace system. While a general (infinite-dimensional) optimal filter theory [1,2] exists for nonlinear systems with Gaussian or non-Gaussian noise, applications rely on (finite-dimensional) suboptimal approximations to the optimal filter for practical implementations. The most widely-studied filters of this kind include the Regularized Particle Filter (RPF) [3, 4] and the Ensemble Square Root Filter (EnSRF) [5]. The latter is an ad-hoc approximation to the Bayes Filter, while the former is rigorously formulated, based upon the Glivenko-Cantelli theorem. By introducing a new global resampling step to the RPF, the EnSRF is proved to approximate the RPF in a special case.
\end{abstract}

Keywords: Ensemble Square Root Filter, Regularized Particle Filter with Langevin Resampling

PACS: $02.50 . \mathrm{Ng}, 05.10 . \mathrm{Gg}, 02.50 . \mathrm{Ey}, 02.50 . \mathrm{Fz}, 02.50 . \mathrm{Cw}$

\section{INTRODUCTION}

For linear systems with Gaussian noise, the problem is completely solved by the Kalman filter (KF). The Ensemble Square Root Filter (EnSRF) originated as a version of the KF for large nonlinear problems and it is now an important data assimilation component of ensemble forecasting. The EnSRF is only strictly valid when all probability distributions are Gaussians, and the forward model is linear. It captures unbiasedness of the mean and the variance of the approximate posterior distribution. Rigorous methods such as the Regularized Particle Filter (RPF) [6, 7] approximate the posterior distribution more accurately without bias in higher moments.

Due to the nonlinearity of the system and the measurement error, sample impoverishment is the most common, key implementation issue of the RPF. The resampling step, which involves drawing samples from the posterior distribution, is designed to solve this problem. The traditional resampling methods, such as Residual, Stratified and Systematic Resampling [8,9], only sample the local function space. If all the particles are located in the tails of the posterior distribution, such resampling schemes will not solve the problem.

The Langevin Algorithm is one of the most famous methods for drawing samples from a target distribution. It has a random forcing term at each pseudo time step and is more likely to break away from a local region of the sampling function space, and is more efficient at sampling the global sampling function space. We begin with the derviation of the Langevin resampling step. Then we state the algorithm of the RPF with Langevin step (RPFLR). Finally the EnSRF is proved to be a special case of our RPFLR for a Gaussian Kernel, with some assumptions.

\section{DERIVATION OF LANGEVIN RESAMPLING}

We consider the state-space system, characterized with additive observation noise as follows:

$$
\begin{aligned}
\text { the state equation: } & x_{k}=f_{k-1}\left(x_{k-1}\right) ; \\
\text { the measurement equation: } & s_{k}=H_{k} x_{k}+v_{k} .
\end{aligned}
$$

Here, the subscript $k$ denotes the time index, $x_{k}$ is the state, $f_{k}(\cdot)$ is the nonlinear system, $s_{k}$ is the measurement, $H_{k}$ is the measurement matrix and $v_{k}$ is the measurement noise. Both $x_{k}$ and $f_{k}$ are $n_{x}$-dimensional, $s_{k}$ and $v_{k}$ are each $n_{s}$-dimensional, while $H_{k}$ has dimension $n_{s} \times n_{x}$. We denote the array of observations $\left\{s_{1}, s_{2}, \ldots, s_{m-1}, s_{m}\right\}$ by $S_{M}$.

Assuming a prior probability density function (pdf), $\pi_{0}\left(x_{0}\right)$, for $x_{0}$ has been provided, we will focus on the filtering problem of determining the posterior pdf $\pi\left(x_{k} \mid S_{k}\right)$ when measurement $s_{k}$ is assimilated at time step $k$. The RPF approximates the posterior pdf $\pi\left(x_{k} \mid S_{k}\right)$ using a continuous kernel density function, by assigning a kernel density function and a weight $w_{k, i}$ to each particle $x_{k, i}$ as shown in eq. (9). Here, we use the Langevin equation to construct a 
random walk for each of our particles $x_{k, i}$, such that the invariant measure of the particles is $\pi\left(x_{k} \mid S_{k}\right)$ :

$$
d \mathbf{X}_{\tau}^{i}=\mathscr{A} D(x, \tau) d \tau+\sqrt{2 \mathscr{A}} d \sigma_{\tau} .
$$

Here, the superscript $i$ is the particle index, $\tau$ is the pseudo-time, $\mathbf{X}_{\tau} \in \mathbb{R}^{n_{x}}$ are the particle states, $\mathscr{A}$ is any positivedefinite self-adjoint matrix $D(x, \tau)$ is the drift vector and $\sigma_{\tau} \in \mathbb{R}^{n_{x}} \sim \mathscr{N}\left(0, I^{n_{x}}\right)$ is a standard Brownian motion. Initially, $\mathbf{X}_{0}^{i}=x_{k, i}$ for all $i=1, \ldots, N$. It is well known that the corresponding evolution for the measurement is the Fokker-Planck equation:

$$
\frac{\partial \pi(x, \tau)}{\partial \tau}=\nabla \cdot[-\mathscr{A} D(x, \tau) \pi(x, \tau)+\mathscr{A} \nabla \pi(x, \tau)] .
$$

Introducing the potential $v$ such that: $D(x, \tau)=-\mathscr{A} \nabla v(x, \tau) \mathrm{r}$, equations (2) and (3) become

$$
\begin{aligned}
d \mathbf{X}_{\tau}^{i} & =-\nabla v(x, \tau) d \tau+\sqrt{2 \mathscr{A}} d \sigma_{\tau}, \\
\frac{\partial \pi(x, \tau)}{\partial \tau} & =\nabla \cdot[\pi(x, \tau) \nabla v(x, \tau)+\mathscr{A} \nabla \pi(x, \tau)] .
\end{aligned}
$$

Using the fact that $\pi(x, \tau) \rightarrow \pi\left(x_{k} \mid S_{k}\right)$ when $\tau \rightarrow \infty$ and combining (4a,b), gives:

$$
d \mathbf{X}_{\tau}^{i}=\mathscr{A} \nabla\left\{\ln \left[\pi\left(x_{k} \mid S_{k}\right)\right]\right\} d \tau+\sqrt{2 \mathscr{A}} d \sigma_{\tau} .
$$

\section{REGULARIZED PARTICLE FILTER WITH A LANGEVIN RESAMPLING STEP (RPFLR)}

The RPFLR for (1a, b) involves the following steps

1. Randomly generate $N$ initial particles $x_{0, i}^{a}$ from a given initial pdf $\pi\left(x_{0}\right)$.

2. For $k=1,2, \cdots$, generate the forecast particles $x_{k, i}^{f}$ using

$$
x_{k, i}^{f}=f_{k}\left(x_{k-1, i}^{a}\right) \quad i=1, \ldots, N .
$$

3. Calculate the mean and variance of the particles using

$$
\hat{x}_{k}^{f}=\frac{1}{N} \sum_{i=1}^{N} x_{k, i}^{f}, \quad P_{k}^{f}=\frac{1}{N-1} \sum_{i=1}^{N}\left(x_{k, i}^{f}-\hat{x}_{k}^{f}\right)\left(x_{k, i}^{f}-i \hat{x}_{k}^{f}\right)^{T}=\frac{1}{N-1} A_{k} A_{k}^{T} .
$$

4. Update the weight $w_{k, i}$ of each particle using

$$
w_{k, i}=\frac{w_{k, i}^{*}}{\sum_{i=1}^{N} w_{k, i}^{*}}, \quad w_{k, i}^{*}=\frac{w_{k-1, i}}{(2 \pi)^{n_{x} / 2}|R|^{1 / 2}} \exp \left(\frac{-\left(\tilde{s}-H_{k} x_{k, i}^{f}\right)^{T} R^{-1}\left(\tilde{s}-H_{k} x_{k, i}^{f}\right)}{2}\right) .
$$

5. Approximate the posterior $\pi\left(x_{k} \mid S_{k}\right)$ by

$$
\pi\left(x_{k} \mid S_{k}\right)=\sum_{i=1}^{N} w_{k, i} K_{h}\left(x_{k}-x_{k, i}^{f}\right), \quad K_{h}(x)=\frac{\exp \left[-1 / 2\left(A^{-1} x / h_{\mathrm{opt}}\right)^{T}\left(A^{-1} x / h_{\mathrm{opt}}\right)\right]}{(2 \pi)^{n_{x} / 2} h_{\mathrm{opt}}^{n_{x}}(\operatorname{det} A)},
$$

Where, $h_{\mathrm{opt}}$ is a scalar, called the bandwidth of the regularized particle filter ${ }^{1}[6,7]$.

The Langevin Resampling step involves:

6. At pseudo time $\tau=0$,

$$
\forall i=1, \ldots, N: \quad \mathbf{x}_{0}^{i}=x_{k, i}^{f}
$$

\footnotetext{
${ }^{1}$ which minimizes the mean integrated error or the mean integrated square error between the approximate and exact posterior distributions
} 
7. For $i=1,2, \ldots, N$, choose an appropriate (small) pseudo time step $\Delta \tau$ and a positive-definite self-adjoint matrix $\mathscr{A}$.

8. Then, for $j=1,2, \ldots$, proceed as follows until the proposal particle is accepted:

(a) Using the Crank-Nicolson numerical scheme for eq. (5), generate a proposal particle $\mathbf{X}_{j}^{i}$ using

$$
\mathbf{X}_{j}^{i}=\mathbf{X}_{j-1}^{i}+\frac{1}{2} \Delta \tau \mathscr{A}\left(\nabla\left\{\ln \left[\pi\left(x_{k}=\mathbf{X}_{j-1}^{i} \mid S_{k}\right)\right]\right\}+\nabla\left\{\ln \left[\pi\left(x_{k}=\mathbf{X}_{j}^{i} \mid S_{k}\right)\right]\right\}\right)+\sqrt{2 \mathscr{A} \Delta \tau} \sigma_{\tau}
$$

where $\sigma_{\tau} \sim \mathscr{N}\left(0, I^{n_{x}}\right)$ is $n_{x}$-dimensional white noise.

(b) The proposal particle is then accepted if $\left|\mathbf{X}_{j}^{i}-\mathbf{X}_{j-1}^{i}\right| \leq \sqrt{2 \mathscr{A} \Delta \tau}$ otherwise it is rejected.

(c) The accepted particle is taken as our analysed particle at time step $k$ :

$$
x_{k, i}^{a}=\mathbf{x}_{j}^{i}
$$

\section{SPECIAL CASE OF RPFLR STEP: ENSRF}

Taking only one stepping the update using eq. (11) of our Langevin Resampling and accept all the proposal particles as the analysed particles, so that:

$$
x_{k, i}^{a}=x_{k, i}^{f}+\frac{\Delta \tau}{2} \mathscr{A} \nabla\left(\left\{\ln \left[\pi\left(x_{k}=x_{k, i}^{f} \mid S_{k}\right)\right]\right\}+\left\{\ln \left[\pi\left(x_{k}=x_{k, i}^{a} \mid S_{k}\right)\right]\right\}\right)+\sqrt{2 \mathscr{A} \Delta \tau} \sigma_{\tau},
$$

where $\sigma_{\tau} \sim \mathscr{N}\left(0, I^{n_{x}}\right)$ is $n_{x}$-dimensional white noise.

Choosing the bandwidth $h_{\mathrm{opt}}=1$, the prior pdf $\pi\left(x_{k} \mid S_{k-1}\right)$ given by the forecasting step is:

$$
\pi\left(x_{k} \mid S_{k-1}\right)=\frac{1}{N} \sum_{i=1}^{N} \mathscr{N}\left(x_{k, i}^{f}, P_{k}^{f}\right) .
$$

This is written in the same spirit as eqns (9). Here, $x_{k, i}^{f}$ and $P_{k}^{f}$ are given by eqns (6) and (7), respectively.

Assuming the prior particles $x_{k, i}^{f}$ are very close to one another in the sampling space, using the axioms of Gaussian pdf addition, the prior pdf $\pi\left(x_{k} \mid S_{k-1}\right)$ in eqn (14) is approximated as:

$$
\pi\left(x_{k} \mid S_{k-1}\right) \approx \mathscr{N}\left(\hat{x}_{k}^{f}, P_{k}^{f}\right)
$$

where $\hat{x}_{k}^{f}$ is calculated using eqn (7).

Substituting the approximate prior pdf (15) and the measurement function (1b) into eqn (7), the posterior pdf $\pi\left(x_{k} \mid S_{k}\right)$ satisfies:

$$
\pi\left(x_{k} \mid S_{k}\right)=\frac{1}{C_{1}} \exp \left\{-\frac{1}{2}\left[\left(x_{k}-\hat{x}_{k}^{f}\right)^{T} P_{k}^{f^{-1}}\left(x_{k}-\hat{x}_{k}^{f}\right)+\left(s_{k}-H x_{k}^{f}\right)^{T} R^{-1}\left(s_{k}-H x_{k}^{f}\right)\right]\right\}
$$

Here,

$$
C_{1}=(2 \pi)^{n_{x}+n_{s}}\left(\operatorname{det} P_{k}^{f}\right)^{1 / 2}(\operatorname{det} R)^{1 / 2} \pi\left(s_{k} \mid S_{k-1}\right)
$$

is a constant. Hence, the key to our Langevin Resampling update will be to evaluate the drift term $\nabla\left\{\ln \left[\pi\left(x_{k} \mid S_{k}\right)\right]\right\}$ in eqn (13). Using eqn (16), this drift term becomes:

$$
\begin{aligned}
\nabla\left\{\ln \left[\pi\left(x_{k} \mid S_{k}\right)\right]\right\} & =-P_{k}^{f^{-1}}\left(x_{k}-\hat{x}_{k}^{f}\right)-H^{T} R^{-1}\left(s_{k}-H x_{k}\right) \\
& =\left(P_{k}^{f^{-1}}+H^{T} R^{-1} H\right)\left[\left(P_{k}^{f^{-1}}+H^{T} R^{-1} H\right)^{-1}\left(H^{T} R^{-1} s_{k}+P_{k}^{f^{-1}} \hat{x}_{k}^{f}\right)-x_{k}\right]
\end{aligned}
$$

Introducing the Kalman gain matrix:

$$
K_{k}=P_{k}^{f} H^{T}\left(H P_{k}^{f} H^{T}+R\right)^{-1}
$$

and applying the matrix inversion lemma ${ }^{2}\left(P_{k}^{f^{-1}}+H^{T} R^{-1} H\right)^{-1}$, equation (17) can be transformed as follows:

$$
\begin{aligned}
\nabla\left\{\ln \left[\pi\left(x_{k} \mid S_{k}\right)\right]\right\} & =\left(P_{k}^{f^{-1}}+H^{T} R^{-1} H\right)\left\{\left[P_{k}^{f}-P_{k}^{f} H^{T}\left(H P_{k}^{f} H^{T}+R\right)^{-1} H P_{k}^{f}\right]\left(H^{T} R^{-1} s_{k}+P_{k}^{f^{-1}} \hat{x^{f}}\right)-x_{k}\right\} \\
& =\left(P_{k}^{f^{-1}}+H^{T} R^{-1} H\right)\left\{\left(\hat{x}_{k}^{f}-x_{k}\right)+K_{k}\left(s_{k}-H \hat{x}_{k}^{f}\right)\right] .
\end{aligned}
$$


Substituting eqn (19) into eqn (13), the analysed particles given by our special case Langevin Resampling step satisfy:

$$
x_{k, i}^{a}=x_{k, i}^{f}+\frac{\Delta \tau}{2} \mathscr{A}\left(P_{k}^{f^{-1}}+H^{T} R^{-1} H\right)\left[2 \hat{x}_{k}^{f}-x_{k, i}^{f}+x_{k, i}^{a}+2 K_{k}\left(s_{k}-H \hat{x}_{k}^{f}\right)\right]+\sqrt{2 \mathscr{A} \Delta \tau} \sigma_{\tau},
$$

where $\sigma_{\tau} \sim \mathscr{N}\left(0, I^{n_{x}}\right)$ is an $n_{x}$ dimensional white noise. It is interesting to note that matrix $A$ and pseudo time step $\Delta \tau$ are operating together as a pre-conditioner on the update step. The requirement is for matrix $A$ to be symmetric and positive-definite. To simplify our Langevin Resampling algorithm (20), we choose

$$
\Delta \tau A=2\left(P_{k}^{f^{-1}}+H^{T} R^{-1} H\right)^{-1} .
$$

With this choice, (20) becomes:

$$
x_{k, i}^{a}=\hat{x}_{k}^{f}+K_{k}\left(s_{k}-H \hat{x}_{k}^{f}\right)+\sqrt{\left(P_{k}^{f^{-1}}+H^{T} R^{-1} H\right)^{-1}} \sigma_{\tau} .
$$

This is the exact form of the EnSRF because the stochastic part of the equation make the analysed particles satisfy the exact Kalman Filter covariance ${ }^{2}$.

\section{CONCLUDING DISCUSSION}

It have been demonstrated that the Regularized Particle Filter is a more rigorous and general filter, compared to Ensemble Square Root Filter. By varying the pre-conditioner $\mathscr{A}$, the performance of our Regularized Particle Filter with Langevin Resampling step can be adjusted. Compared to the Ensemble Filters, the RPFLR has the capability of sampling the global function space of posterior pdfs. Further work is need to compare to other particle and kernel methods and the RPFLR regarding the efficienc y of the sampling.

\section{ACKNOWLEDGMENTS}

This publication was based on work supported in part by Award No KUK-C1-013-04, made by King Abdullah University of Science and Technology (KAUST).

\section{REFERENCES}

1. A. H. Jazwinski, Stochastic Processes and Filtering Theory, Academic Press, 1970.

2. G. Kallianpur, Stochastic Filtering Theory, No. 13 in Applications of Mathematics, Springer Verlag, 1980.

3. A. Doucet, N. de Freitas, and N. Gordon, Sequential Monte Carlo Methods in Practice, Springer New York, USA, 2001.

4. B. Ristic, S. Arulampalam, and N. Gordon", Beyond the Kalman Filter: Particle Filters for Tracking Applications, Artech House Publishers, 2004.

5. J. L. Anderson, Monthly Weather Review 129, 2884-2903 (2001).

6. L. Devroye, A Course in Density Estimation, Birkhauser, 1987.

7. B. W. Silverman, Density Estimation for Statistics and Data Analysis, Chapman \& Hall, 1986.

8. G. Kitagawa, Journal of Computational and Graphical Statistics 5, 1-25 (1996).

9. D. Whitley, Stat. Comput. 4, 65-85 (1994).

\footnotetext{
${ }^{2}$ Using the matrix inversion lemma: $\left(P_{k}^{f^{-1}}+H^{T} R^{-1} H\right)^{-1}=P_{k}^{f}-P_{k}^{f} H^{T}\left(H P_{k}^{f} H^{T}+R\right)^{-1} H P_{k}^{f}=\left(I-K_{k} H^{T}\right) P_{k}^{f}$
} 



\section{RECENT REPORTS}

11/10 Nonlinear Correction to the Euler Buckling Formula for Compressible Cylinders

De Pascalis

Destrade

Goriely

12/10 Nonlinear Morphoelastic Plates I: Genesis of Residual Stress

McMahon

Goriely

Tabor

13/10 Nonlinear Morphoelastic Plates II: Exodus to Buckled States

McMahon

Goriely

Tabor

14/10 Analysis of Brownian dynamics simulations of reversible biomolecular reactions

Lipkova

Zygalakis

Chapman

Erban

15/10 Travelling waves in hyperbolic chemotaxis equations

Xue

Hwang

Painter

Erban

16/10 The Physics and Mechanics of Biological Systems

Goriely

Moulton

17/10 Crust formation in drying colloidal suspensions

Style

Peppin

18/10 A Mathematical Model of Tumor-Immune Interactions

Robertson-Tessi

El-Kareh

Goriely

19/10 Elastic cavitation, tube hollowing, and differential growth in plants and biological tissues

Goriely

Moulton

Vandiver

20/10 Asymptotic expressions for the nearest and furthest dislocations

Hall in a pile-up against a grain boundary

21/10 Cardiac electromechanics: the effect of contraction model on the mathematical problem and accuracy of the numerical scheme

Pathmanathan

Chapman

Gavaghan

Whiteley

22/10 Fat vs. thin threading approach on GPUs: application to stochastic simulation of chemical reactions

Klingbeil

Erban

Giles

Maini

23/10 Asymptotic analysis of a system of algebraic equations arising in dislocation theory

Hall

Chapman

Ockendon

25/10 Preconditioning for Allen-Cahn Variational Inequalities with Non- Blank Local Constraints Sarbu

Stoll

26/10 On an evolution equation for sand dunes Ellis Fowler 
28/10 An a posteriori error analysis of a mixed finite element Galerkin Memon approximation to second order linear parabolic problems Nataraj

Pani

29/10 A Priori Error Estimates for Semidiscrete Finite Element Approx-

Goswami imations to Equations of Motion Arising in Oldroyd Fluids of Or-

Pani der One

30/10 The Landau-de Gennes theory of nematic liquid crystals: Uniaxiality versus Biaxiality

31/10 The Radial-Hedgehog Solution in Landau-de Gennes' theory

Majumdar

32/10 Nonlinear instability in flagellar dynamics: a novel modulation mechanism in sperm migration?

Gadelha

Gaffney

Smith

Kirkman-Brown

33/10 Error bounds on block GaussSeidel solutions of coupled multi-

Whiteley physics problem

Gillow

Tavener

Walter

34/10 A random projection method for sharp phase boundaries in lattice

Reis Boltzmann simulations

Dellar

Copies of these, and any other OCCAM reports can be obtained from:

Oxford Centre for Collaborative Applied Mathematics

Mathematical Institute

24 - 29 St Giles'

Oxford

OX1 3LB

England

www.maths.ox.ac.uk/occam 